\title{
A BURNS-KRANTZ TYPE THEOREM FOR DOMAINS WITH CORNERS
}

\author{
LUCA BARACCO, DMITRI ZAITSEV, AND GIUSEPPE ZAMPIERI
}

\section{INTRODUCTION}

In their pioneering paper BK94, BURNs and KRANTz have established a boundary version of the classical CARTAN's uniqueness theorem:

If $f$ is holomorphic self-map of a smoothly bounded strongly pseudoconvex domain $D \subset$ $\mathbb{C}^{N}$ such that $f(z)=z+o\left(|z-p|^{3}\right)$ as $z \rightarrow p$ for some $p \in \partial D$, then $f(z) \equiv z$.

Furthermore, they give an example showing that the exponent 3 in the above statement cannot be decreased. More recent results of this kind are due to VLACCI-TAURASO VT98 and Bracci-Vlacci-Tauraso BVT00 in one and to Huang H95 and GentiliMigliorini GM97 in several complex variables. A basic global assumption in these results was that the given holomorphic map is a self-map of the given bounded domain.

The goal of this paper is twofold. First, to give purely local boundary uniqueness results for maps defined only on one side as germs at a boundary point and hence not necessarily sending any domain to itself and also under the weaker assumption that $f(z)=z+$ $o\left(|z-p|^{3}\right)$ holds only for $z$ in a proper cone in $D$ with vertex $p$. Such results have no analogues in one complex variable in contrast to the situation when a domain is preserved. And second, to extend the above results from boundaries of domains to submanifolds of higher codimension. Here the usual replacement for a one-sided neighborhood of $M$ is a wedge with the edge $M$. The notion of strong pseudoconvexity can be also extended to this situation (see \$2 for more details). We prove:

Theorem 1.1. Let $M \subset \mathbb{C}^{N}, N \geq 2$, be a generic submanifold at a point $p \in M$ and $f$ be a germ at $p$ of a holomorphic self-map of a strongly pseudoconvex wedge with edge $M$ at $p$ such that

$$
f(z)=z+o\left(|z-p|^{3}\right)
$$

as $z$ approaches $p$ nontangentially. Then $f(z) \equiv z$. Moreover, we can restrict the way $z$ approaches $p$ by requiring $z$ to belong to a cone with vertex $p$, only depending only on the domain of definition of $f$, which is properly contained in the given wedge.

\footnotetext{
${ }^{0}$ Dipartimento di Matematica, Università di Padova, via Belzoni 7, 35131 Padova, Italy, baracco@math.unipd.it, zaitsev@math.unipd.it, zampieri@math.unipd.it
} 
Note that the map $f$ is not assumed to have any limit at points of the edge $M$ other than $p$ (though it has nontangential limits almost everywhere by a result of FORSTNERIČ Fo92] and, in case it has a continuous boundary value, it is not assumed to send $M$ into itself. The authors of this paper are not aware of any other rigidity results for maps of this kind. Here the known method based on the LEMPERT theory of extremal discs for the Kobayashi metric [L81a, L81b, L82] cannot be applied. Instead, our approach makes use of the theory of stationary discs recently developed by TUMANOV [T01].

We conclude by giving a consequence of Theorem 1.1 in the hypersurface case which is a "local" and "nontangential" version of corresponding results of [BK94, H95]:

Corollary 1.2. Let $M \subset \mathbb{C}^{N}, N \geq 2$, be a strongly pseudoconvex hypersurface at a point $p \in M$ and $f$ be a germ at $p$ of a holomorphic self-map of the pseudoconvex side such that $f(z)=z+o\left(|z-p|^{3}\right)$ as $z$ approaches $p$ nontangentially. Then $f(z) \equiv z$.

In fact we shall obtain Theorem [1.1 and Corollary 1.2 as special cases of the more general Theorem 2.2 below, where the source and the target of $f$ may be different even as germs at $p$.

\section{Preliminaries AND generalizations}

Let $M \subset \mathbb{C}^{N}$ be a smooth submanifold. Recall that $M$ is called generic in $\mathbb{C}^{N}$ if its tangent space at each point spans $\mathbb{C}^{N}$ over complex numbers. Denote by $T^{*} \mathbb{C}^{N}$ the cotangent bundle of $\mathbb{C}^{N}$ (regarded as the space of all $(1,0)$ forms on $\mathbb{C}^{N}$ ) and, for every $p \in M$, by $N_{p} M:=T_{p} \mathbb{C}^{N} / T_{p} M$ the normal and by $N_{p}^{*} M \subset T_{p}^{*} \mathbb{C}^{N}$ the conormal spaces to $M$ in $\mathbb{C}^{N}$, where $N_{p}^{*} M$ consists of all 1 -forms $\varphi \in T_{p}^{*} \mathbb{C}^{N}$ such that $\left.\operatorname{Re} \varphi\right|_{T_{p} M}=0$. Recall that the (vector-valued) Levi form of $M$ at $p \in M, L_{p}: T_{p}^{1,0} M \times T_{p}^{1,0} M \rightarrow N_{p} M \otimes \mathbb{C}$ is the (unique) hermitian form such that the equality

$$
L_{p}\left(X_{p}, Y_{p}\right)=\frac{1}{2 i}[X, \bar{Y}]_{p} \quad \bmod T_{p}^{1,0} M \oplus T_{p}^{0,1} M
$$

holds for all vector fields $X$ and $Y$ in $T^{1,0} M$, where $X_{p}$ stands for the evaluation of the vector field $X$ at $p$. The Levi cone $C_{p}=C_{p} M \subset N_{p} M$ is the convex hull of all vectors $L_{p}(v, v)$ for $v \in T_{p}^{1,0} M \backslash\{0\}$ and the dual Levi cone is $C_{p}^{*} M:=\left\{\xi \in N_{p}^{*} M:\left.\xi\right|_{C_{p} M}>\right.$ $0\}$. The notion of strong pseudoconvexity can be extended to submanifolds of higher codimension as follows. The submanifold $M \subset \mathbb{C}^{N}$ is strongly pseudoconvex at $p \in M$ (see e.g. TH83, Fo91, T01]) if $C_{p}^{*} M \neq \emptyset$, i.e. if $\xi(L(u, v))$ is positive definite for some conormal $\xi$. It follows that $M$ is strongly pseudoconvex at $p$ if and only if there exists a strongly pseudoconvex (real) hypersurface in $\mathbb{C}^{N}$ that contains a neighborhood of $p$ in $M$ (see e.g. [Fo91]).

We now introduce the notion of a strongly pseudoconvex wedge with edge $M$ at $p$ which generalizes strongly pseudoconvex sides of hypersurfaces and which is relevant for the rigidity result given by Theorem 2.2 . For open cones $\Gamma, \Gamma^{\prime} \subset \mathbb{R}^{n}$, we write $\Gamma \ll \Gamma^{\prime}$ if $\bar{\Gamma} \backslash\{0\} \subset \Gamma^{\prime}$ and say that $\Gamma$ is properly contained in $\Gamma^{\prime}$. More generally, a cone $\Gamma \subset \mathbb{R}^{n}$ 
is said to be proper in an open subset $D \subset \mathbb{R}^{n}$ if it is properly contained in another cone $\Gamma^{\prime} \subset \mathbb{R}^{n}$ such that a neighborhood of 0 in $\Gamma^{\prime}$ is contained in $D$. By a wedge with edge $M$ at $p$ in the direction of an open cone $\Gamma \subset N_{p} M$ we shall understand here any domain $W \subset \mathbb{C}^{N}$ such that for all open cones $\Gamma^{\prime}, \Gamma^{\prime \prime} \subset N_{p} M$ with $\Gamma^{\prime} \ll \Gamma \ll \Gamma^{\prime \prime}$ and for every sufficiently small neighborhood $U$ of $p$ in $\mathbb{C}^{N}$, one has:

$$
\begin{gathered}
(M \cap U)+\left(\Gamma^{\prime} \cap U\right) \subset W ; \\
(M \cap U)+\left(\Gamma^{\prime \prime} \cap U\right) \text { contains a neighborhood of } p \text { in } W,
\end{gathered}
$$

where the normal space $N_{p} M$ is identified with any fixed complementary subspace to $T_{p} M$ in $\mathbb{C}^{N}$. It is easy to see that the given definition is independent of the choice of such a complement and also of the choice of local coordinates. Conditions (2.1) and (2.2) give estimates on the shape of $W$ from inside and from outside respectively and clearly depend only on the intersection of $W$ with an arbitrarily small neighborhood of $p$ in $\mathbb{C}^{N}$.

We call $\Gamma$ the directional cone of $W$ at $p$. We say that the wedge $W$ is strongly pseudoconvex at $p$ if its directional cone $\Gamma$ satisfies the following:

$$
\begin{gathered}
L_{p}(v, v) \in \Gamma \text { for some vector } v \in T_{p}^{1,0} M ; \\
\xi \text { is positive on } \bar{\Gamma} \backslash\{0\} \text { for some covector } \xi \in C_{p}^{*} M .
\end{gathered}
$$

Here $L_{p}$ denotes the Levi form of $M$ at $p$ as above. As in the above definition of a wedge, also here (2.3) and (2.4) are estimates on the shape of $\Gamma$ from inside and from outside respectively. In particular, it follows from (2.4) that $C_{p}^{*} M$ is nonempty and hence $M$ is automatically strongly pseudoconvex in the above sense. An important class of examples can be obtained as follows.

Example 2.1. Let $\rho_{1}, \ldots, \rho_{d}, 1 \leq d \leq N-1$, be strictly plurisubharmonic functions in a neighborhood of $p$ in $\mathbb{C}^{N}$, vanishing at $p$ and satisfying $\partial \rho_{1} \wedge \cdots \wedge \partial \rho_{d} \neq 0$. Then

$$
W:=\left\{\rho_{1}<0, \ldots, \rho_{d}<0\right\}
$$

is a strongly pseudoconvex wedge at $p$ with edge $M:=\left\{\rho_{1}=\ldots=\rho_{d}=0\right\}$. In particular, an one-sided neighborhood of a real hypersurface is a strongly pseudoconvex wedge if and only if it is the strongly pseudoconvex side in the usual sense.

Another important class is given by Siegel domains of 2nd kind (see [P69]) that are strongly pseudoconvex wedges whose edges are their Shilov boundaries.

The following is a generalization of Theorem 1.1 .

Theorem 2.2. Let $M \subset \mathbb{C}^{N}, N \geq 2$, be a generic submanifold through a point $p, U$ and $V$ be strongly pseudoconvex wedges with edge $M$ at $p$ and $f$ be a germ at $p$ of a holomorphic map between $U$ and $V$ with $f(z)=z+o\left(|z-p|^{3}\right)$ as $z$ approaches $p$ nontangentially. Then $f(z) \equiv z$. 
It will follow from the proof that one has the same statement under the weaker assumptions that $U$ satisfies only (2.1) and (2.3) (for some cone $\Gamma \subset N_{p} M$ ) and $V$ satisfies only (2.2) and (2.4) (for a possibly different cone $\left.\widetilde{\Gamma} \subset N_{p} M\right)$. Also, we can weaken the assumptions about the asymptotics of $f$ by asking that $f(z)=z+o\left(|z-p|^{2}\right)$ and $\langle\xi, f(z)\rangle=\langle\xi, z\rangle+o\left(|z-p|^{3}\right)$ for at least one covector $\xi$ such that (2.4) holds. We shall write $U$ (resp. $V$ ) for wedges with edge $M$ in the statements below that will be used for the source (resp. target) wedge in Theorem 2.2

\section{One-DIMENSIONAL CASE}

One of the key points in the proofs of mentioned rigidity results in BK94] and [H95] is the reduction to the one-dimensional situation, where $D=\Delta$ is the unit disc in $\mathbb{C}$. The latter case is therefore of particular importance. We begin with a new elementary proof of the following result due to [BK94, H95]:

Proposition 3.1. Let $f: \Delta \rightarrow \Delta$ be a holomorphic self-map with $f(z)=z+o\left(|z-1|^{3}\right)$ as $z \rightarrow 1$ in $\Delta$. Then $f(z) \equiv z$.

Proof. By the classical Fatou's theorem, $f$ has an $L^{\infty}$ boundary value function on $\partial \Delta$ that we denote also by $f$. Since $f(z) \in \bar{\Delta}$ for all $z \in \bar{\Delta}$, we have

$$
\operatorname{Re}\left(\bar{z} \frac{z-f(z)}{|z-1|^{4}}\right) \geq 0, \quad z \in \partial \Delta .
$$

We write $K_{\varepsilon}(1)$ for the disc with center 1 and radius $\varepsilon>0$. Then

$$
\begin{aligned}
\operatorname{Re} \int_{\left\{\theta: e^{i \theta} \notin K_{\varepsilon}(1)\right\}} e^{-i \theta} \frac{e^{i \theta}-f\left(e^{i \theta}\right)}{\left|e^{i \theta}-1\right|^{4}} d \theta=\operatorname{Im} & \int_{\partial \Delta \backslash K_{\varepsilon}(1)} z^{2} \bar{z} \frac{z-f(z)}{(z-1)^{4}} \frac{d z}{z} \\
& =\operatorname{Im} \int_{\partial K_{\varepsilon}(1) \cap \Delta} z \frac{z-f(z)}{(z-1)^{4}} \frac{d z}{z} \rightarrow 0, \quad \varepsilon \rightarrow 0
\end{aligned}
$$

where the second equality holds because the function under the integral extends holomorphically to $\Delta$ and the convergence to 0 is a consequence of the estimate $f(z)=$ $z+o\left(|z-1|^{3}\right)$. Hence we must have in (3.1) the equality almost everywhere on $\partial \Delta$ proving $f(z) \equiv z$ as required.

The next result, for which we give a self-contained proof, generalizes Proposition 3.1 .

Proposition 3.2. Let $f: \Delta \rightarrow \Delta$ be a holomorphic self-map, $z_{k} \in \Delta$ a sequence converging nontangentially to 1 such that $f\left(z_{k}\right)=z_{k}+o\left(\left|z_{k}-1\right|^{3}\right)$ as $k \rightarrow \infty$. Then $f(z) \equiv z$.

Proof. We begin by deriving a special case of the classical Julia's Theorem following [R80]. For $a \in \Delta$, consider the automorphism $\varphi_{a}(z):=(a-z) /(1-\bar{a} z)$ of $\Delta$ interchanging $a$ and 0 . Then we have

$$
\left|\varphi_{f(a)}(f(z))\right| \leq\left|\varphi_{a}(z)\right|
$$


for all $a, z \in \Delta$ as a consequence of the Schwarz lemma. We next use the identity

$$
1-\left|\varphi_{a}(z)\right|^{2}=\frac{\left(1-|a|^{2}\right)\left(1-|z|^{2}\right)}{|1-\bar{a} z|^{2}}
$$

that can be verified by easy computation (see [R80, Theorem 2.2.2]). Then (3.2 3.3) imply

$$
\frac{|1-\overline{f(a)} f(z)|^{2}}{1-|f(z)|^{2}} \leq \frac{1-|f(a)|^{2}}{1-|a|^{2}} \frac{|1-\bar{a} z|^{2}}{1-|z|^{2}} .
$$

Setting $a=z_{k}$ and taking the limit we obtain the estimate

$$
\frac{|1-f(z)|^{2}}{1-|f(z)|^{2}} \leq \frac{|1-z|^{2}}{1-|z|^{2}}
$$

which is the desired special case of Julia's Theorem.

The second part of the proof closely follows the proof of Lemma 2.1 in [H95]. An easy computation shows that the harmonic function

$$
\xi(z):=\operatorname{Re}\left(\frac{1+z}{1-z}-\frac{1+f(z)}{1-f(z)}\right)
$$

is nonnegative in view of (3.5). Furthermore, we have $\left|\left(f\left(z_{k}\right)-z_{k}\right) /\left(1-z_{k}\right)\right|<1$ for $k$ sufficiently large and hence, using the geometric series expansion,

$$
\frac{1+f\left(z_{k}\right)}{1-f\left(z_{k}\right)}=\frac{\left(1+z_{k}+f\left(z_{k}\right)-z_{k}\right) /\left(1-z_{k}\right)}{1-\left(f\left(z_{k}\right)-z_{k}\right) /\left(1-z_{k}\right)}=\frac{1+z_{k}}{1-z_{k}}+o\left(\left|z_{k}-1\right|\right), \quad k \rightarrow \infty .
$$

Hence $\xi\left(z_{k}\right)=o\left(\left|z_{k}-1\right|\right)$ as $k \rightarrow \infty$. In view of the Hopf lemma, the latter fact is only possible if $\xi(z) \equiv 0$ and therefore $f(z) \equiv z$ as required.

\section{STATIONARY DISCS}

We review basic fact from the TuMANOV's theory of stationary discs in higher codimension extending the classical LEMPERT's theory for strictly (linearly) convex domains. Recall that a continuous mapping $\Phi: \bar{\Delta} \rightarrow \mathbb{C}^{N}$, where $\Delta$ is the unit disc in $\mathbb{C}$, is called an analytic disc attached to $M$ if $\left.\Phi\right|_{\Delta}$ is holomorphic and $\Phi(\partial \Delta) \subset M$. In this paper we shall consider only smooth analytic discs. In his celebrated paper [L81a], LEMPERT gave a characterization of those analytic discs attached to a boundary of a strictly convex domain, for which the maximum of the Kobayashi distance is attained, in terms of meromorphic lifts of its boundary values. The discs admitting such lifts were called stationary. More recently, Tumanov [T01] extended this notion to higher codimension. In his terminology, an analytic disc $\Phi$ attached to $M$ is stationary if there exists a nonzero holomorphic map (a lift) $\Phi^{*}: \bar{\Delta} \backslash\{0\} \rightarrow T^{*} \mathbb{C}^{N}$ such that $\zeta \Phi^{*}(\zeta) \in \mathcal{O}(\Delta) \cap \mathcal{C}(\bar{\Delta})$ and $\Phi^{*}(\zeta) \in N_{\Phi(\zeta)}^{*} M$ for $\zeta \in \partial \Delta$. In contrast to the full space of attached analytic discs, the space of stationary discs (satisfying certain inequalities) has finite dimension provided $M$ is sufficiently nondegenerate (see Theorem 4.1 below). 
In order to have an explicit description of stationary discs, it will be convenient to write $M$ near a point $p \in M$ in the form

$$
M=\{\rho(x+i y, w)=0\}=\{y=h(x, w)\}, \quad \rho(x+i y, w)=h(x, w)-y,
$$

where coordinates $(z, w)=(x+i y, w) \in \mathbb{C}^{d} \times \mathbb{C}^{n}=\mathbb{C}^{N}$ vanishing at $p$ are chosen such that $h(0)=0$ and $h^{\prime}(0)=0$. In order to have a description of all holomorphic (and meromorphic) lifts of an attached analytic disc $\Phi$, it will be convenient, following [T88, T01, to introduce "partial holomorphic lifts" of $\Phi$ to $T^{*} \mathbb{C}^{N}$ (or their collections forming a matrix) for which only the "z-components" are holomorphic. More precisely, to every analytic disc $\Phi$ sufficiently small (e.g. in $\mathcal{C}^{\infty}(\bar{\Delta})$ ) which is attached to $M$ (given in the form (4.1)), one can associate, by solving a Bishop's equation, a unique smooth real invertible $d \times d$ matrix function $G(\zeta)$ on $\partial \Delta$ such that $G(1)=$ id and $G(\zeta) \rho_{z}(\Phi(\zeta))$ extends holomorphically from $\partial \Delta$ to $\Delta$, where $\rho_{z}:=\left(\rho_{z_{1}}, \ldots, \rho_{z_{d}}\right)$ is the gradient with respect to the chosen coordinates. (More precisely, the function $G(\zeta)$ here is normalized at $\zeta=1$ rather than $\zeta=0$ as in [T01 and, hence, differs from it by an invertible constant matrix factor.) We now state TUMANOV's existence and uniqueness result for stationary discs in the following slightly adapted for our purposes form:

Theorem 4.1 (Tumanov). Let $M \subset \mathbb{C}^{N}$ be a smooth generic submanifold through a point $p$. Then for every fixed $c^{0} \in \mathbb{R}^{d}$ such that the Levi form of $M$ at $p$ is nondegenerate in the direction of the conormal $c^{0} \partial \rho$, every fixed $\varepsilon>0$ and every data $(\lambda, c, v) \in \mathbb{C}^{d} \times$ $\mathbb{R}^{d} \times \mathbb{C}^{n}$ sufficiently close to $\left(0, c^{0}, 0\right)$, there exists a unique smooth stationary disc $\Phi(\zeta)=$ $(z(\zeta), w(\zeta)), \zeta \in \bar{\Delta}$, with $\Phi(1)=p$ and $w^{\prime}(1)=v$ whose lift $\Phi^{*}$ is of the form

$$
\Phi^{*}(\zeta)=(\operatorname{Re}(\lambda \zeta+c)) G(\zeta) \partial \rho(\Phi(\zeta))
$$

and such that $w(\zeta)=\alpha(\zeta)+\beta(\zeta)$ with $\alpha$ linear and $\|\beta\|_{\mathcal{C}^{\infty}}<\varepsilon\|v\|$.

Since Theorem 4.1] is not explicitly stated in this form in [T01], we provide here a proof, essentially following the arguments of [T01, §4-5].

Proof. Let $M$ be given near $p=0$ by (4.1) and let $\Phi: \bar{\Delta} \rightarrow \mathbb{C}^{N}$ be a sufficiently small stationary disc for $M$ with a lift $\Phi^{*}: \bar{\Delta} \backslash\{0\} \rightarrow T^{*} \mathbb{C}^{N}$. Denote by $G(\zeta)$ the invertible $d \times d$ matrix function on $\partial \Delta$ as described above such that $G(1)=$ id and $G(\zeta) \rho_{z}(\Phi(\zeta))$ extends holomorphically to $\Delta$. Observe that $G(\zeta)$ is arbitrarily close to the constant identity provided $\Phi$ is arbitrarily small. We can then write

$$
\Phi^{*}(\zeta)=\nu(\zeta) G(\zeta)\left(\rho_{z}(\Phi(\zeta)), \rho_{w}(\Phi(\zeta))\right), \quad \zeta \in \partial \Delta
$$

where $\nu: \partial \Delta \rightarrow \mathbb{R}^{d}$ is a real vector function. By definition of the lift, $\zeta \Phi^{*}(\zeta)$ extends holomorphically to $\Delta$. Recall that the construction of $G$ required $\Phi$ to be small. We can also further require that the inverse $S(\zeta):=\left(G(\zeta) \rho_{z}(\Phi(\zeta))\right)^{-1}$ exists and extends holomorphically to $\Delta$. Multiplying $\zeta \Phi^{*}(\zeta)$ by $S(\zeta)$ on the right and using (4.3) we conclude that the product $\zeta \nu(\zeta)$ must also extend holomorphically to $\Delta$. Since $\nu$ is valued in $\mathbb{R}^{d}$, 
expanding into the Fourier series on $\partial \Delta$, we conclude that $\nu(\zeta)=\operatorname{Re}(\lambda \zeta+c)$ for suitable vectors $\lambda \in \mathbb{C}^{d}, c \in \mathbb{R}^{d}$. In particular, $\Phi^{*}(\zeta)$ is always of the form (4.2).

We now explore, under what conditions, an attached analytic disc $\Phi$ with a lift $\Phi^{*}$ given by (4.2) is stationary, i.e. when does $\zeta \Phi^{*}(\zeta)$ extend holomorphically to $\Delta$. Since

$$
2 \zeta \operatorname{Re}(\lambda \zeta+c)=\zeta(\lambda \zeta+\bar{\lambda} \bar{\zeta}+2 c)=\lambda \zeta^{2}+\bar{\lambda}+2 c \zeta, \quad \zeta \in \partial \Delta,
$$

obviously has a holomorphic extension to $\Delta$ and since $G(\zeta) \rho_{z}(\Phi(\zeta))$ extends holomorphically to $\Delta$ by the construction, then

$$
\zeta(\operatorname{Re}(\lambda \zeta+c)) G(\zeta) \rho_{z}(\Phi(\zeta)), \quad \zeta \in \partial \Delta,
$$

also has the same extension property. Hence it remains to find $\Phi(\zeta)$ such that the " $w$ component"

$$
\zeta(\operatorname{Re}(\lambda \zeta+c)) G(\zeta) \rho_{w}(\Phi(\zeta)), \quad \zeta \in \partial \Delta,
$$

has the extension property. The fact that $\Phi(\zeta)=(z(\zeta), w(\zeta))$ is attached to $M$ with $\Phi(1)=0$ can be expressed via the Bishop's equation

$$
\operatorname{Re} z(\zeta)=-T_{1}(h(\operatorname{Re} z(\zeta), w(\zeta))), \quad \zeta \in \partial \Delta,
$$

so that $w(\zeta)$ with $w(1)=0$ can be seen as an independent parameter and $z(\zeta)$ is uniquely determined from it by (4.7) and the condition $z(1)=0$.

We next assume, without loss of generality, that $h(x, w)$ is further normalized as

$$
h(x, w)=\left(w^{*} A_{j} w\right)_{j}+o\left(|(x, w)|^{2}\right), \quad(x, w) \rightarrow 0,
$$

where Hermitian $n \times n$ matrices $A_{1}, \ldots, A_{d}$ represent the ( $\mathbb{R}^{d}$-valued) Levi form of $M$ at 0 , and that the corresponding normalization holds also for the derivatives:

$$
\rho_{w}(z, w)=h_{w}(z, w)=\left(\bar{A}_{j} \bar{w}\right)_{j}+o(|(x, w)|), \quad(x, w) \rightarrow 0,
$$

where we think of $L_{w}$ as a $d \times n$ matrix (we write $*$ for the conjugate transposed matrix). The next step consists of substituting (4.9) into (4.6) and writing $G(\zeta)=\mathrm{id}+G_{0}(\zeta)$ and

$$
\sum_{j=1}^{d}\left(\operatorname{Re}\left(\lambda_{j} \zeta+c_{j}\right)\right) A_{j}=(\mathrm{id}-\zeta X)^{*} B(\mathrm{id}-\zeta X), \quad \zeta \in \partial \Delta,
$$

for suitable $n \times n$ complex matrices $B=B(\lambda, c), X=X(\lambda, c)$. To show the existence of $B$ and $X$, we set $P=P(\lambda, c):=\sum_{j} \lambda_{j} A_{j}, Q=Q(\lambda, c):=\sum_{j} c_{j} A_{j}$ and rewrite (4.10) as

$$
\zeta P+\bar{\zeta} P^{*}+2 Q=B-\zeta B X-\bar{\zeta} X^{*} B+X^{*} B X
$$

leading to the system

$$
P^{*} X^{2}+2 Q X+P=0, \quad 2 Q=B+X^{*} B X .
$$

Recall that we consider $(\lambda, c)$ in a neighborhood of $\left(0, c^{0}\right)$ such that $P\left(0, c^{0}\right)=0$ and $Q\left(0, c^{0}\right)$ is invertible. Then the system (4.12) can be solved for $(X, B)$ near $\left(0, B^{0}\right)$ by the implicit function theorem with $B^{0}=2 Q\left(0, c^{0}\right)$ being invertible. 
We now look for $w(\zeta)$ in the form

$$
w(\zeta)=w_{0}+\zeta(\mathrm{id}-\zeta X)^{-1} \sigma(\zeta)
$$

where $\sigma(\zeta)$ is an unknown function and $w_{0}$ is uniquely determined by the condition $w(1)=$ 0 . Note that, if $(X, B)$ is sufficiently close to $\left(0, B^{0}\right)$, the matrix (id $\left.-\zeta X\right)$ is invertible for $\zeta \in \bar{\Delta}$. Substituting (4.13) (and the corresponding solution $z(\zeta)$ of (4.7)) into (4.9) and using (4.10) and the property of $G(\zeta)$, we conclude that the expression in (4.6) can be written as

$$
\zeta(\operatorname{Re}(\lambda \zeta+c))\left(\bar{A}_{j} \bar{w}_{0}\right)_{j}+(\mathrm{id}-\bar{\zeta} \bar{X})^{*} \bar{B} \overline{\sigma(\zeta)}+o(\|\sigma\|), \quad \zeta \in \partial \Delta,
$$

where the norm of $\sigma$ in the last term is understood in any Hölder space $\mathcal{C}^{\alpha}, 0<\alpha<1$. The first term always extends holomorphically to $\Delta$ in view of (4.4). Hence the holomorphic extendibility of (4.6) is equivalent to that of

$$
(\text { id }-\bar{\zeta} \bar{X})^{*} \bar{B} \overline{\sigma(\zeta)}+o(\|\sigma\|), \quad \zeta \in \partial \Delta .
$$

Furthermore, since the matrix factor in (4.15) is invertible and holomorphic in $\zeta$, our condition is equivalent to the antiholomorphic extendibility of $\sigma(\zeta)+o(\|\sigma\|)$. By taking a Cauchy transform $K$ given by the Cauchy integral formula up to a constant and using the fact that $\sigma$ is holomorphically extendible, we obtain an equation $\sigma(\zeta)=a+K(o(\|\sigma\|))$, where $a \in \mathbb{C}^{n}$ is a parameter that can be chosen to be $\sigma(1)$. The latter equation can be solved by the implicit function theorem in any Hölder class $\mathcal{C}^{k, \alpha}$ for any sufficiently small $a=\sigma(1)$ and any data $(\lambda, c)$ sufficiently close to $\left(0, c^{0}\right)$. It can be now seen from the construction and the implicit function theorem that the solution $w(\zeta)$ given by (4.13) defines a smooth transformation $(\lambda, c, a) \mapsto\left(\lambda, c, w^{\prime}(1)\right)$ sending $\left(0, c^{0}, 0\right)$ into itself and being locally invertible there. This shows the existence and uniqueness of a small solution $w(\zeta)$ with given data $\lambda, c$ and $v=w^{\prime}(1)$ having the required properties. The proof is complete.

Note that, by the construction in [T01, for $c$ fixed and $\lambda, v$ arbitrarily small, the difference $\Phi^{*}(\zeta)-\xi$ is also arbitrarily small on $\partial \Delta$, where $\xi:=\Phi^{*}(1)$. We shall say that a stationary disc $\Phi$ has a small lift $\Phi^{*}$ in the direction of a conormal $\xi \in N_{p}^{*} M$ if $\Phi(1)=p$, $\Phi^{*}(1)=\xi$ and both $\Phi(\zeta)-p, \Phi^{*}(\zeta)-\xi$ are small in the norm $\mathcal{C}^{\infty}(\partial \Delta)$. Then the uniqueness part of Theorem 4.1 implies that, if $\xi \in C_{p}^{*}$, all stationary discs with small lifts in the direction $\xi$ are given by Theorem 4.1 with sufficiently small data $\lambda, v$.

We now show that the assumption (2.3) in for a wedge with edge $M$ implies that the corresponding stationary discs are contained in the wedge.

Lemma 4.2. Let $U$ be a wedge with edge $M$ at $p$ whose directional cone is $\Gamma$ and $v_{0} \in$ $T_{p}^{1,0} M$ be such that $L_{p}\left(v_{0}, v_{0}\right) \in \Gamma$. Then, for any $\xi \in C_{p}^{*}$, any neighborhood of the origin contains an open subset of parameters $\lambda, v$ for which the corresponding stationary discs $\Phi$ with small lifts in the direction $\xi$ satisfy $\Phi(\Delta) \subset U$. Moreover, for any sector $S \subset \Delta$ with 
vertex 1 and sufficiently small parameters $\lambda, v$, the images $\Phi(S)$ are contained in a fixed proper cone in $U$ with vertex $p$.

Proof. By Theorem 4.1 there exist stationary discs $\Phi(\zeta)=(z(\zeta), w(\zeta))$ with arbitrarily small lifts in the direction $\xi$ with $w^{\prime}(1) \in \mathbb{C} v_{0}$. Since also $w(\zeta)=\alpha(\zeta)+\beta(\zeta)$ with $\alpha$ and $\beta$ satisfying the conclusion of Theorem 4.1 and since $\Phi$ is attached to $M$, the derivatives $\Phi^{\prime}(\zeta)$ will be arbitrarily close to a multiple of $v_{0}$ for all $\zeta \in \bar{\Delta}$. Choose any conormal $c \partial \rho(p) \in N_{p}^{*} M$ with $c d \rho\left(p ; L_{p}\left(v_{0}, v_{0}\right)\right)>0$, where $c \in \mathbb{R}^{d}$ and $\rho$ is the defining function of $M$ near $p$. Then, for $\Phi$ as above, the function $c \rho \circ \Phi$ is plurisubharmonic in $\Delta$ and is zero on the boundary $\partial \Delta$. By the maximum principle, $c \rho(\Phi(\zeta)) \leq 0$ for all $\zeta \in \Delta$. By choosing finitely many conormals $c_{1} \partial \rho, \ldots, c_{s} \partial \rho$ such that

$$
L_{p}\left(v_{0}, v_{0}\right) \in \bigcap_{j}\left\{c_{j} d \rho(p)>0\right\} \subset \subset \Gamma,
$$

we conclude that $\Phi(\Delta) \subset \cap_{j}\left\{c_{j} \rho<0\right\} \subset U$ for $\Phi$ sufficiently small. The last conclusion follows from the Hopf lemma applied to suitable small perturbations of $\rho$.

Lemma 4.3. Let $\Phi_{0}$ be a stationary disc with sufficiently small lift in the direction $\xi \in$ $N_{p}^{*} M$. Then, for stationary discs $\Phi$ arbitrarily close to $\Phi_{0}$ in the direction $\xi$, the union of their boundaries $\Phi(\partial \Delta)$ contains open subsets in $M$ arbitrarily close to $p$.

Proof. By Theorem 4.1, the discs $\Phi$ are in one-to-one correspondence with the parameters $\lambda \in \mathbb{C}^{d}, v \in \mathbb{C}^{n}$. We then consider the correspondence

$$
(\lambda, v) \underset{\psi}{\longmapsto}\left(\Phi^{\prime}(1),\left(\Phi^{*}\right)^{\prime}(1)\right) \underset{\pi}{\mapsto} \Phi^{\prime}(1), \quad \mathbb{C}^{d} \times \mathbb{C}^{n} \rightarrow T_{(p, \xi)} N^{*} M \rightarrow T_{p} M .
$$

Since $M$ is strongly pseudoconvex, $\Phi$ has defect 0 , i.e. its lift $\Phi^{*}$ cannot be chosen holomorphic. By [T01, Proposition 3.9], $\psi$ is injective and hence immersive on a dense subset. Since $\operatorname{dim}_{\mathbb{R}} N^{*} M=2(n+d), \psi$ is also submersive on a dense subset of its domain of definition. Then $\pi \circ \psi$ is clearly submersive on the same subset and the required statement follows.

Lemmas 4.2 and 4.3 imply:

Corollary 4.4. In the setting of Lemma 4.2 the union of images $\Phi(\Delta)$ of the stationary discs $\Phi$ in the direction $\xi$ arbitrarily close to $\Phi_{0}$, contains smooth generic submanifolds of $\mathbb{C}^{N}$ arbitrarily close to $p$.

\section{Strongly CONVEX hypersurfaces PASsing through Stationary Discs}

Following LEMPERT [L81a] we call an analytic disc $\Phi: \bar{\Delta} \rightarrow \mathbb{C}^{N}$ stationary for a bounded domain with smooth boundary $\Omega \subset \mathbb{C}^{N}$ if it is stationary for $M=\partial \Omega$ and the lift $\Phi^{*}$ can be chosen such that it does not vanish on $\partial \Delta$. Our goal here is to construct, for each sufficiently small stationary disc $\Phi$, a smooth strongly convex domain for which $\Phi$ is also stationary with the same lift. 
Proposition 5.1. Suppose that a wedge $V$ with edge $M$ at $p$ and a conormal $\xi \in C_{p}^{*}$ satisfies (2.4). Then there exist a biholomorphic change of coordinates in $\mathbb{C}^{N}$, a neighborhood $B(p)$ of $p$ in $\mathbb{C}^{N}$ and, for every stationary disc $\Phi$ with sufficiently small data $\lambda, v$ as in Theorem 4.1 and $\Phi^{*}(1)$ close to $\xi$, a strongly convex (with respect to the new coordinates) bounded domain $\Omega \subset \mathbb{C}^{N}$ such that

(i) $V \cap B(p) \subset \Omega$;

(ii) $M \cap B(p) \subset \partial \Omega$;

(iii) $\left.\Phi^{*}(\zeta)\right|_{T_{\Phi(\zeta)} \partial \Omega}=0$ and $\Phi^{*}(\zeta) \neq 0$ for all $\zeta \in \partial \Delta$.

In particular, $\Phi$ is stationary also for $\Omega$ with the same lift $\Phi^{*}$.

Proof. In the vector notation we have, for every stationary disc $\Phi$ as above, $\Phi^{*}(\zeta)=$ $c(\zeta) \partial \rho(\Phi(\zeta))$ for $\zeta \in \partial \Delta$, where $c(\zeta)$ is a real vector and $\rho$ is the defining function of $M$ as in (4.1). If the data $\lambda, v$ are sufficiently small, it follows from Theorem 4.1 that $\Phi: \bar{\Delta} \rightarrow \mathbb{C}^{N}$ is a smooth embedding. Hence we can find a smooth extension $\widetilde{c}$ of $c$ from $\Phi(\partial \Delta) \cong \partial \Delta$ to a neighborhood of $p$ in $M$. The neighborhood can be chosen uniformly for all $\Phi$ as above. Moreover, since $\Phi^{*}(\zeta)$ is arbitrarily close to $\xi$ and $\xi$ satisfies (2.4), also the extension $\tilde{c} \partial \rho$ can be chosen to have these properties. Then one can see that the real hypersurface defined by $\widetilde{\rho}:=\widetilde{c} \rho+C\|\rho\|^{2}$ for a sufficiently large constant $C>0$ near $p$ can be extended to the boundary of a domain $\Omega$ as required.

In the situation of Proposition 5.1, we can take advantage of LEMPERT's theory L81a, L81b, in particular, of the fact that stationary discs coicide with geodesics for the Kobayashi metric and also that the latter are regular up to the boundary.

\section{A DiRECT PROOF OF ThEOREM 2.2 IN A WEAKER FORM}

Here we give a direct proof of the statement of Theorem 2.2. where the asymptotics $f(z)=z+o\left(|z-p|^{3}\right)$ is assumed for any $z$ in the wedge rather than in a proper cone. We begin by a uniqueness result for stationary discs in smooth strongly convex domains.

Proposition 6.1. Let $\Omega \subset \mathbb{C}^{N}$ be a smooth strongly convex bounded domain and $\Phi: \bar{\Delta} \rightarrow$ $\bar{\Omega}$ be a stationary disc for $\Omega$. Let $\widetilde{\Phi}: \Delta \rightarrow \Omega$ be a holomorphic map satisfying $\widetilde{\Phi}(\zeta)=$ $\Phi(\zeta)+o\left(|\zeta-1|^{3}\right)$ as $\zeta \rightarrow 1$ in $\Delta$. Then $\widetilde{\Phi} \equiv \Phi$.

Proof. Since $\Phi$ is stationary for $\Omega$, there exists a (smooth) lift $\Phi^{*}: \bar{\Delta} \backslash\{0\} \rightarrow T^{*} \mathbb{C}^{N}$ such that $\Phi^{*}(\zeta) \in N_{\Phi(\zeta)}^{*} \partial \Omega \backslash\{0\}$ for $\zeta \in \partial \Delta$ and $\zeta \Phi^{*}(\zeta)$ is holomorphic in $\Delta$. By the classical Fatou's theorem, $\widetilde{\Phi}$ has an $L^{\infty}$ boundary value on $\partial \Delta$ that we also denote by $\widetilde{\Phi}$. Since $\Omega$ is strongly convex, we can choose $\Phi^{*}$ such that

$$
\operatorname{Re}\left\langle\Phi^{*}(\zeta), \frac{\Phi(\zeta)-\widetilde{\Phi}(\zeta)}{|\zeta-1|^{4}}\right\rangle \geq 0
$$

for almost all $\zeta \in \partial \Delta$, where $\langle\cdot, \cdot\rangle$ denotes the standard pairing. Furthermore, since $\Omega$ is strongly convex, for almost every $\zeta \in \partial \Delta$, we have the equality in (6.1) if and only 
if $\Phi(\zeta)=\widetilde{\Phi}(\zeta)$. Since $\Phi$ is smooth up to the boundary of $\Delta$ and because of the given estimate for $\widetilde{\Phi}$, we can repeat the arguments of the proof of Proposition 3.1 to obtain

$$
\operatorname{Re} \int_{\left\{\theta: e^{i \theta} \notin K_{\varepsilon}(1)\right\}}\left\langle\Phi^{*}\left(e^{i \theta}\right), \frac{\Phi\left(e^{i \theta}\right)-\widetilde{\Phi}\left(e^{i \theta}\right)}{\left|e^{i \theta}-1\right|^{4}}\right\rangle d \theta \rightarrow 0, \quad \varepsilon \rightarrow 0 .
$$

Hence we must have the equality in (6.1) almost everywhere on $\partial \Delta$ which proves $\Phi \equiv \widetilde{\Phi}$ on $\Delta$ as required.

We now turn to the proof of Theorem 2.2 in the weaker form mentioned above:

Proposition 6.2. The conclusion of Theorem 2.2 holds if the hypothesis on the asymptotics of $f$ at $p$ holds without restrictions on the way of approaching $p$.

Proof. By Lemma 4.2, for any $\xi \in C_{p}^{*}$, there exists an open set of stationary discs $\Phi$ with arbitrarily small lifts in the direction $\xi$ such that $\Phi(\Delta) \subset U$. In view of Corollary 4.4, it is enough to show that $f \circ \Phi \equiv \Phi$ on $\Delta$ for each disc $\Phi$ as above. For $\Phi$ fixed, let $B(p)$ and $\Omega$ be given by Proposition [5.1. Since $f(z) \rightarrow p$ as $z \rightarrow p$ and since $\Phi$ can be taken arbitrarily small, we may assume that $\Phi(\Delta) \subset V \cap B(p) \subset \Omega$. Since $\Phi$ is smooth up to the boundary, we have the estimate $f(\Phi(\zeta))=\Phi(\zeta)+o\left(|\zeta-1|^{3}\right)$ and hence we are in the situation of Proposition 6.1 with $\widetilde{\Phi}:=f \circ \Phi$ that yields $\widetilde{\Phi} \equiv \Phi$ as required.

\section{UNIQUENESS FOR IMAGES OF STATIONARY DISCS}

In order to prove Theorem 2.2 as it stands we shall need results of LEMPERT L81a, L81b, L82 for stationary discs and geodesics and a result of HUANG [H95] on boundary uniqueness for holomorphic self-maps of the disc $\Delta$. The main consequence can be stated as follows.

Proposition 7.1. Let $\Omega$ and $\Phi$ be as in Proposition 6.1 and $\widetilde{\Phi}: \Delta \rightarrow \Omega$ be a holomorphic map satisfying $\widetilde{\Phi}\left(z_{k}\right)=\Phi\left(z_{k}\right)+o\left(\left|z_{k}-1\right|^{3}\right)$ for some sequence $z_{k}$ in $\Delta$ converging to 1 nontangentially. Then $\widetilde{\Phi} \equiv \Phi$.

Proof. Since $\Phi$ is stationary for $\partial \Omega$, there exists a holomorphic retraction $\pi: \Omega \rightarrow \Delta$ such that $\pi \circ \Phi=\mathrm{id}_{\Delta}$ (see [L81a, L81b, L82]) and $\pi$ is smooth up to the boundary of $\Omega$. Hence $\pi \circ \widetilde{\Phi}$ is a holomorphic self-map of $\Delta$ satisfying $\pi\left(\widetilde{\Phi}\left(z_{k}\right)\right)=z_{k}+o\left(\left|z_{k}-1\right|^{3}\right)$. Then $\pi \circ \widetilde{\Phi}=\mathrm{id}$ by Proposition 3.2. Since both $\pi$ and $\widetilde{\Phi}$ are holomorphic, we have

$$
K_{\Delta}\left(\pi\left(\widetilde{\Phi}\left(\zeta_{1}\right)\right), \pi\left(\widetilde{\Phi}\left(\zeta_{2}\right)\right)\right) \leq K_{\Omega}\left(\widetilde{\Phi}\left(\zeta_{1}\right), \widetilde{\Phi}\left(\zeta_{2}\right)\right) \leq K_{\Delta}\left(\zeta_{1}, \zeta_{2}\right),
$$

where $K_{D}$ denotes the Kobayashi distance in a domain $D$. Since $\pi \circ \widetilde{\Phi}=\mathrm{id}$, the first and the last distances in (7.1) are the same. We conclude that $K_{\Omega}\left(\widetilde{\Phi}\left(\zeta_{1}\right), \widetilde{\Phi}\left(\zeta_{2}\right)\right)=K_{\Delta}\left(\zeta_{1}, \zeta_{2}\right)$ holds for all $\zeta_{1}, \zeta_{2} \in \Delta$ and hence $\widetilde{\Phi}$ is a complex geodesic of $\Omega$. Since $\Omega$ is strongly convex and smooth, it follows from [L81a, L81b] that $\widetilde{\Phi}$ is smooth up to the boundary of $\Delta$. The regularity of $\Phi$ and $\widetilde{\Phi}$ on $\bar{\Delta}$ together with Taylor's formula yields the improved estimate 
$\widetilde{\Phi}(\zeta)=\Phi(\zeta)+o\left(|\zeta-1|^{3}\right)$ for $\zeta \rightarrow p$ without any restriction on the way of approaching $p$. The required conclusion follows from Proposition 6.1. Alternatively, one can use at this last stage uniqueness results for complex geodesics.

\section{Proofs of Theorems $1.2-2.2$}

The proof of Theorem 2.2 is obtained by repeating the proof of Proposition 6.2 and using Proposition 7.1 instead of Proposition 6.1. Note that we enter the hypotheses of Proposition 7.1 in view of the last conclusion of Lemma 4.2. Theorem 1.1 is a special case of Theorem 2.2 with $U=V$.

\section{REFERENCES}

[A89] Abate, M. - Iteration theory of holomorphic maps on taut manifolds. Research and Lecture Notes in Mathematics. Complex Analysis and Geometry. Mediterranean Press, Rende, 1989.

[BVT00] Bracci, F.; Vlacci, F.; Tauraso, R. - Identity Principles for Commuting Holomorphic Self-Maps of the Unit Disc. J. Math. Anal. Appl., to appear.

[BK94] Burns, D.M.; Krantz, S.G. - Rigidity of holomorphic mappings and a new Schwarz lemma at the boundary. J. Amer. Math. Soc. 7 (1994), no. 3, 661-676.

[H95] Huang, X. - A boundary rigidity problem for holomorphic mappings on some weakly pseudoconvex domains. Canad. J. Math. 47 (1995), no. 2, 405-420.

[Fo91] Forstnerič, F. - Mappings of strongly pseudoconvex Cauchy-Riemann manifolds. Several complex variables and complex geometry, Part 1 (Santa Cruz, CA, 1989), 59-92, Proc. Sympos. Pure Math. 52, Part 1, Amer. Math. Soc., Providence, RI, 1991.

[Fo92] Forstnerič, F. - Admissible boundary values of bounded holomorphic functions in wedges. Trans. Amer. Math. Soc. 332 (1992), no. 2, 583-593.

[GM97] Gentili, G.; Migliorini, S. - A boundary rigidity problem for holomorphic mappings. Proceedings of the Third International Workshop on Differential Geometry and its Applications and the First German-Romanian Seminar on Geometry (Sibiu, 1997). Gen. Math. 5 (1997), 161-174.

[L81a] Lempert, L. - La métrique de Kobayashi et la représentation des domaines sur la boule. Bull. Soc. Math. France 109 (1981), no. 4, 427-474.

[L81b] Lempert, L. - Intrinsic distances and holomorphic retracts. Complex analysis and applications '81 (Varna, 1981), 341-364, Publ. House Bulgar. Acad. Sci., Sofia, 1984.

[L82] Lempert, L - Holomorphic retracts and intrinsic metrics in convex domains. Anal. Math. 8 (1982), no. 4, 257-261.

[P69] Pyateskii-Shapiro, I.I. - Automorphic functions and the geometry of classical domains. Translated from the Russian. Mathematics and Its Applications, Vol. 8. Gordon and Breach Science Publishers, New York-London-Paris, 1969.

[R80] Rudin, W. - Function theory in the unit ball of $C^{n}$. Grundlehren der Mathematischen Wissenschaften [Fundamental Principles of Mathematical Science], 241. Springer-Verlag, New York-Berlin, 1980.

[T88] Tumanov, A.E. - Extension of CR-functions into a wedge from a manifold of finite type. Mat. Sb. (N.S.) 136 (178) (1988), no. 1, 128-139; translation in Math. USSR-Sb. 64 (1989), no. $1,129-140$. 
[T93] Tumanov, A.E. - On the propagation of extendibility of CR functions. Complex analysis and geometry (Trento, 1993), 479-498, Lecture Notes in Pure and Appl. Math., 173, Dekker, New York, 1996.

[T01] Tumanov, A.E. - Extremal discs and the regularity of CR mappings in higher codimension. Amer. J. Math. 123 (2001), no. 3, 445-473.

[TH83] Tumanov, A. E.; Henkin, G. M. - Local characterization of holomorphic automorphisms of Siegel domains. Funktsional. Anal. i Prilozhen. 17 (1983), no. 4, 49-61.

[VT98] Vlacci, F.; Tauraso, R. - Rigidity at the Boundary for Holomorphic Self-Maps of the Unit Disc. Complex Variables Theory Appl. 45 (2001), no. 2, 151-165. 